\section{Simulation of the Fisher-Zeaman multiple-look attention theory}

\author{
MARY ANN FISHER \\ University of Maryland Baltimore County \\ Baltimore, Maryland 21228
}

Description. The program produces response strings for statistical subjects acting in accord with the assumptions of the attention-retention theory of twochoice visual discrimination learning and memory described by Fisher and Zeaman (1973), in userspecified problem sequences. The theory generalizes the Zeaman and House (1963) attentional model of discrimination learning to include multiple looking with parallel processing of dimensions and cuesignificance feedback features, and adapts the multicomponent multiprocess model of human memory proposed by Atkinson and Shiffrin (1969) to the retention of learned items. A fundamental feature of the theory is the distinction developed by Atkinson and Shiffrin between structural features or fixed capacities of the memory system and the control processes or strategies available for their use in a particular task. The memory-control processes available in the program are the rehearsal-buffer size for each dimension and the probability of entering a new item into the rehearsal buffer to displace an old item. These two control processes are particularly useful in describing the results of discrimination learning analogues of continuous paired associate experiments. The program has been used to simulate results of experiments in discrimination learning and memory including dimensional shift, reversal, cue-blocking, recombination of cues, tests of component retention, proactive and retroactive interference, concurrent problems, and one- and two-dimensional item rehearsal capacity (see Fisher \& Zeaman, 1973; McBane, 1976; Zieger, 1974, for these and other examples). The user specifies the trial-by-trial and session-by-session structure of the experiment to be simulated, the parameter value to be used, and type and magnitude of criterion for a trials-to-criterion design.

Input. The program requests the following input. For each experiment to be simulated: the number of dimensions, the number of trials per session, the number of sessions per subject, the number of subjects to be run, and criterion run information, where applicable.

The preparation of this manuscript was supported in part by Grant M-1099 from the National Institute of Mental Health, U. S. Public Health Service. Requests for reprints should be sent to the author at the Department of Psychology, University of Maryland Baltimore County, 5401 Wilkens Avenue, Baltimore, Maryland 21228.
For each group of subjects: short-term memory decay rate, reward and nonreward learning rate parameters for perceptual and response learning, rehearsal strategy parameter. For each trial: cue-identification numbers for each dimension on the positive and negative stimuli, intertrial interval. For each dimension: maximum rehearsal-buffer size, initial salience, total number of cues in the experiment.

Output. A list of current parameter values is printed out for each group of subjects. The final attentional and cue strengths are printed out for each subject, followed by the string of overt correct responses and errors for that subject.

User Options. The simulated experiment may specify a fixed number of sessions (or trials) or may specify one of two types of criterion runs. When a criterion run is specified, each subject continues only until he meets the criterion. In the first kind of criterion run, the subject is terminated after a run of a specified number of consecutive correct overt responses. In the second type, the subject is terminated after making a specified number of correct responses within a session. The user may specify initial cue strengths for each cue of the experiment or may permit these strengths to be randomly generated and assigned to cues by the program.

Limitations. Current limitations are fairly arbitrary and easily changed. The program now is designed to run up to 60 trials in each of four sessions with up to 55 cues on each of up to five dimensions. There is no restriction on number of subjects.

Computer and Language. Two versions of the program are available. The first is written in PL/I and has been run on the IBM 360 . The second, more limited version is written in CPS, a subset of the PL/I language adapted for use on an IBM 2741 remote terminal in communication with an IBM 360.

Availability. The documented program is available free of charge from the author at the Department of Psychology, University of Maryland Baltimore County, 5401 Wilkens Avenue, Baltimore, Maryland 21228.

\section{REFERENCES}

Atkinson, R. C., \& Shiffrin, R. M. Human memory, a proposed system and its control processes. In K. W. Spence and J. T. Spence (Eds.), The psychology of learning and motivation: Advances in research and theory (Vol. 2). New York: Academic Press, 1969.

Fisher, M. A., \& ZeAman, D. An attention-retention theory of retardate discrimination learning. In N. R. Ellis (Ed.), International review of research in mental retardation (Vol. 6). New York: Academic Press, 1973.

McBane, B. M. Rehearsal capacity and dimensional independence in retardates. Journal of Experimental Child Psychology, 1976, 22, 216-228. 
Zeaman, D., \& House, B. J. The role of attention in retardate discrimination learning. In N. R. Ellis (Ed.), Handbook of mental deficiency. New York: McGrawHill, 1963.
Zieger, W. M. The role of attentional dynamics upon cueblocking and incidental learning in retarded children: Some theoretical comparisons. Unpublished doctoral dissertation, University of Connecticut, 1974. 